\title{
On the biophysics of cathodal galvanotaxis in rat prostate cancer cells: Poisson-Nernst-Planck equation approach
}

\author{
Przemysław Borys
}

Received: 31 October 2011/Revised: 8 March 2012/Accepted: 13 March 2012/Published online: 31 March 2012

(C) The Author(s) 2012. This article is published with open access at Springerlink.com

\begin{abstract}
Rat prostate cancer cells have been previously investigated using two cell lines: a highly metastatic one (Mat-Ly-Lu) and a nonmetastatic one (AT-2). It turns out that the highly metastatic Mat-Ly-Lu cells exhibit a phenomenon of cathodal galvanotaxis in an electric field which can be blocked by interrupting the voltage-gated sodium channel (VGSC) activity. The VGSC activity is postulated to be characteristic for metastatic cells and seems to be a reasonable driving force for motile behavior. However, the classical theory of cellular motion depends on calcium ions rather than sodium ions. The current research provides a theoretical connection between cellular sodium inflow and cathodal galvanotaxis of Mat-Ly-Lu cells. Electrical repulsion of intracellular calcium ions by entering sodium ions is proposed after depolarization starting from the cathodal side. The disturbance in the calcium distribution may then drive actin polymerization and myosin contraction. The presented modeling is done within a continuous one-dimensional Poisson-Nernst-Planck equation framework.
\end{abstract}

Keywords Cathodal galvanotaxis .

Poisson-Nernst-Planck equation · Mat-Ly-Lu · Motility · Prostate cancer · Metastasis

Dedicated to the memory of my wife Marzena, mother of Nina and Sonia, who supported me in this work for the last time.

\section{P. Borys $(\bowtie)$}

Department of Physical Chemistry and Technology of Polymers, Section of Physics and Applied Mathematics, Silesian University of Technology, 44-100 Ks. M. Strzody 9, Gliwice, Poland e-mail: Przemyslaw.Borys@polsl.pl

\section{Introduction}

Prostate cancer cells have been investigated for many years by the group of Prof. Djamgoz at Imperial College (Grimes et al. 1995; Siwy et al. 2003; Djamgoz et al. 2001; Mycielska and Djamgoz 2004). One of the most important results concerning these cells is the finding that the voltagegated sodium channel (VGSC) activity correlates with their metastatic potential. Blocking the VGSCs of the highly metastatic Mat-Ly-Lu cells using tetrodotoxin decreases their metastatic potential, having no effect on the weakly metastatic AT-2 cells (Grimes et al. 1995). Another result, of crucial importance for the presented research, concerns the examination of the motile properties of these cells. Mat-Ly-Lu cells exhibit sensitivity to the external electric field in a phenomenon of cathodal galvanotaxis, which is anodal and much less pronounced in the nonmetastatic AT-2 cells (Djamgoz et al. 2001).

Galvanotaxis was later discovered in further cancer cell lines: the MDA-MB-231 breast cancer line (Fraser et al. 2005; Isbilen et al. 2006) and the A549 lung cancer line (Yan et al. 2009). The lung cancer A549 exhibits cathodal galvanotaxis, as in the case of the Mat-Ly-Lu prostate cancer, while the breast cancer MDA-MB-231 exhibits anodal galvanotaxis, in contrast to Mat-Ly-Lu. This orientation difference will be addressed in the "Results and Discussion" section of the present paper. Apart from cancer cells, galvanotaxis has also been discovered in a number of other cells, as enumerated, for example, by Mycielska and Djamgoz (2004).

The galvanotactic response of a cell makes use of a crawling motion whose molecular mechanism depends on reorganization of the cytoskeleton in response to changes in the internal calcium concentration (Mycielska and Djamgoz 2004; Bray 2001). This consists of four subsequent stages: 
(1) protrusion growth, (2) protrusion adhesion, (3) cell contraction, and (4) detachment of the rear side of the cell. Protrusion growth occurs due to actin filament polymerization, which is regulated by the protein gelsolin and $\mathrm{pH}$. Gelsolin, in the presence of calcium ions, caps the fast-growing barbed ends of f-actin, inhibiting their polymerization and severing them. Low $\mathrm{pH}$ values facilitate actin self-assembly by compensating the anionic character of the monomers (Wang et al. 1989). The contraction of the cell is mediated by myosin, which contracts using adenosine triphosphate (ATP) in the presence of calcium ions (Bray 2001). Within such a framework, directed locomotion may arise after the calcium ion concentration at the rear side of the cell increases, slowing down actin polymerization and causing cell detachment by contraction, while the concentration at the front side decreases, improving actin polymerization (Mycielska and Djamgoz 2004), as illustrated in Fig. 1.

The importance of calcium ions in the motility process leads to consideration of possible calcium pathways into the cytoplasm (Fig. 2): voltage-gated calcium channels (VGCCs), $\mathrm{Na}^{+} / \mathrm{Ca}^{2+}$ exchangers (NCX), plasma membrane $\mathrm{Ca}^{2+}$ ATP-ases (PMCAs), leakage channels, internal rearrangements by calcium "waves," or eventually,

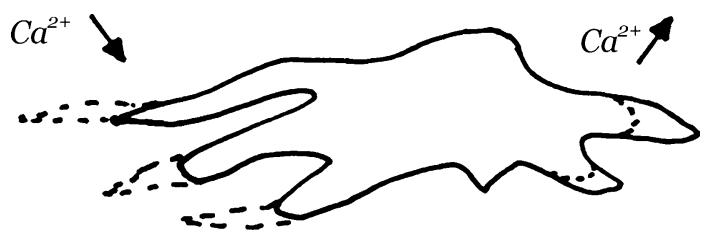

Fig. 1 The calcium-mediated crawling process of a cell. A decrease in calcium concentration drives actin polymerization while the increase in calcium reduces polymerization, and causes actin truncation and myosin contraction calcium release (uptake) from internal stores (Monteith et al. 2007; Carafoli and Brini 2007). It would seem reasonable to expect, for example, that blocking calcium inflow through the VGCCs should have a profound impact on the cellular motion of Mat-Ly-Lu cells. However, this turns out not to be the case, and VGCC activity is not observed in these cells (Djamgoz et al. 2001). Instead, the cellular motion decreases after blockade of VGSCs by tetrodotoxin (TTX) (Djamgoz et al. 2001). A similar effect of VGSC blockade was found in the (anodal) galvanotaxis of MDA-MB-231 breast cancer (Fraser et al. 2005; Isbilen et al. 2006). This finding is consistent with the relation of the VGSCs to the metastatic potential of prostate cancer cells, but the relation between the VGSCs and the cathodal cellular motion remains to be elucidated.

One of the classical options for calcium inflow in galvanotaxis (which I critically discuss in the next sections) is through leakage pathways, which are expected to result in a greater contribution to the anodal than to the cathodal part in the presence of a direct-current (DC) field (Cooper and Keller 1984). This would happen because the anodal part of the cell is polarized in the DC field to contain an attractive negative charge while the cathodal part contains a repulsive positive charge (Fig. 3). However, such a mechanism shows no direct relation to the VGSCs, and it remains unclear why it should stop working in Mat-Ly-Lu cells after TTX administration.

This lack of relation to VGSCs could be overcome by considering the NCX exchanger activity (Fig. 2), which stops removing $\mathrm{Ca}^{2+}$ from the cell in the presence of $\mathrm{Na}^{+}$ (i.e., after VGSC activation) (Hilge et al. 2007). However, NCX does not manifest in Mat-Ly-Lu cells (Djamgoz et al. 2001). Another option could then be $\mathrm{pH}$-driven facilitation of actin polymerization due to $\mathrm{pH}$ lowering after the VGSC

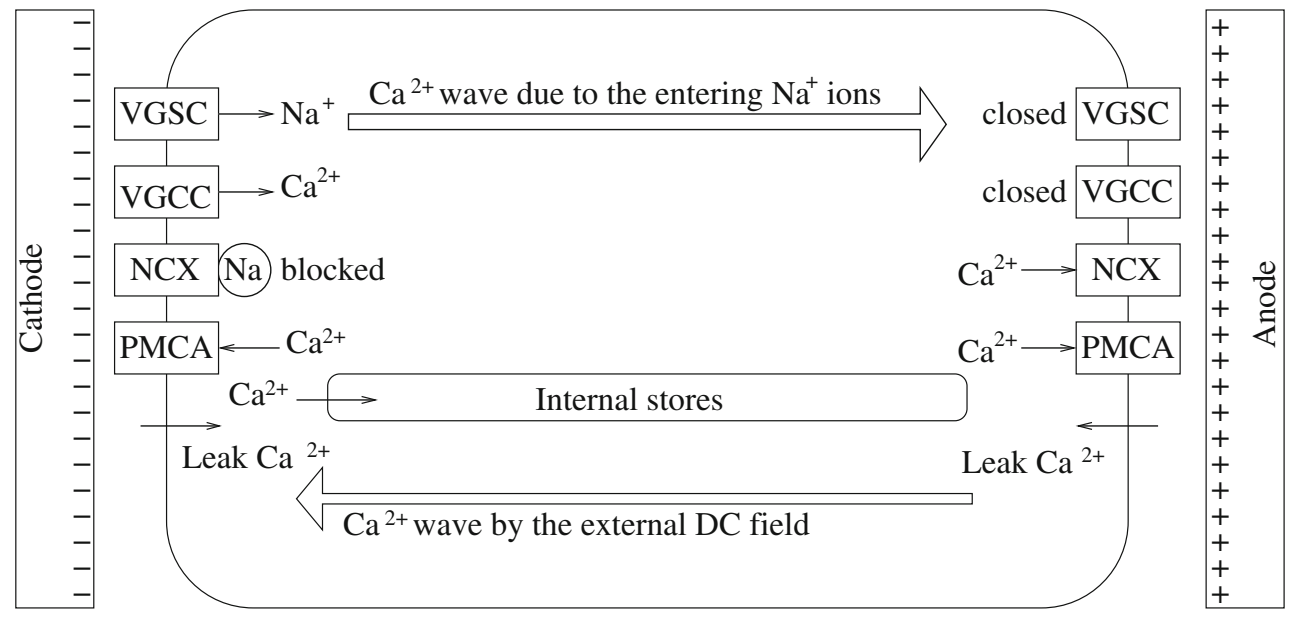

Fig. 2 Calcium flow in the cell, relevant for the galvanotaxis of general cells. VGCCs and NCXs do not manifest in Mat-Ly-Lu cells (Djamgoz et al. 2001) 


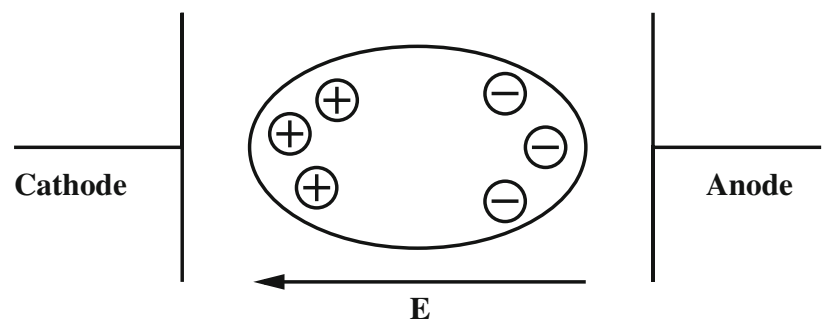

Fig. 3 Polarization of ions inside the cell in the presence of an external electric field

activity (Carrithers et al. 2007) and reduced calcium release from internal stores due to their sensitivity to $\mathrm{pH}$ lowering (Willoughby et al. 2001). However, since the depolarization should eventually spread over the whole cell membrane, this effect could only be transient.

A new effect, supported by informal modeling done on a small amount of discrete ions, was recently proposed by me during the 2008 Gliwice Scientific Meetings (Borys 2008). I suggested that the concentration difference in the $\mathrm{Ca}^{2+}$ ions between the cathodal and anodal sides of the cell may result from electrical repulsion of intracellular $\mathrm{Ca}^{2+}$ ions by $\mathrm{Na}^{+}$ions entering through the VGSCs on the cathodal side. The VGSCs on the cathodal side should activate more easily compared with the anodal side because of the membrane potential reduction in the presence of a DC field (Fig. 3). Before the depolarization wave can reach the anodal part of the membrane, an electric field gradient builds on the cathodal side, forcing calcium ions to move towards the anode. Then, after the anodal part opens its VGSCs as the membrane depolarization continues, the ions cannot be turned back to the cathode because it already contains a large positive charge (larger than the anodal part). Such an effect also explains the galvanotactic response of the AT-2 cells, which do not upregulate the VGSCs and consequently cannot produce a disturbance in the $\mathrm{Ca}^{2+}$ distribution by this mechanism. The importance of timing between the cathode and anode also displays how the NCX activity (in cells other than Mat-Ly-Lu) and VGSC-mediated $\mathrm{pH}$ interactions can become directional.

In the present research, the idea of $\mathrm{Ca}^{2+}$ repulsion by entering $\mathrm{Na}^{+}$ions is investigated in a formal continuous model using the one-dimensional Poisson-Nernst-Planck (PNP) equation approach. The coefficients for this equation (diffusion coefficients, concentrations) were found through a literature search. The boundary conditions mirror the physical DC field. The model system initially spends some time at rest to obtain ionic equilibrium, then $\mathrm{Na}^{+}$ions are injected to the cathodal compartment, mimicking depolarization. Calcium propagation curves are obtained, revealing an increase in the anodal concentration and a decrease in the cathodal concentration.

\section{Methods}

The PNP equation

The problem of ionic diffusion is complex because, whenever the ion concentration changes, the electric field distribution changes as well. The electric field in turn influences the forces acting on the ions. Such a process cannot be described by normal diffusion. A drift-diffusion model is required, which implements the forcing of the ions by the electric potential. The mass flux in the driftdiffusion model in its simplest form can be described by the equation (Berg 1982)

$J=-D \frac{\partial c}{\partial x}+v_{\mathrm{d}} c$,

where $c$ is the species concentration, $x$ is the spatial coordinate, and $v_{\mathrm{d}}$ is the drift velocity, obtained from the Langevin equation (Risken 1989)

$m a=-f v+F+\xi(t)$,

where $f$ is the damping coefficient, $F$ is the external force, and $\xi$ is the random force. Assuming a high-friction limit (Risken 1989), i.e., that the term $m a$ is small compared with other terms in (2), and calculating the average velocity (the drift velocity), one obtains

$v_{\mathrm{d}}=\frac{F}{f}$.

In case of a force generated by an electric potential, the following relation holds for $F$ (Griffiths 1999):

$F=-q \frac{\mathrm{d} \varphi}{\mathrm{d} x}=-z e \frac{\mathrm{d} \varphi}{\mathrm{d} x}$,

where $q$ is the ionic charge described as a multiple $z$ of the elementary charge $e$.

To obtain the drift velocity one must substitute (4) into (3), taking into account the Einstein relation for the diffusion coefficient (Berg 1982) (i.e., $D=k T / f$, where $D$ is the diffusion coefficient, $k$ is the Boltzmann constant, and $T$ is temperature). The final result reads

$v_{\mathrm{d}}=-\frac{D z e}{k T} \frac{\mathrm{d} \varphi}{\mathrm{d} x}=-\frac{D z F}{R T} \frac{\mathrm{d} \varphi}{\mathrm{d} x}$,

where $F$ is the Faraday constant and $R$ is the gas constant. Concluding, the flux reads

$J=-D \frac{\partial c}{\partial x}-\frac{D z F c}{R T} \frac{\mathrm{d} \varphi}{\mathrm{d} x}$.

Constructing a mass balance equation leads to the NernstPlanck equation (Coalson and Kurnikova 2007; Kurnikova et al. 1999; Rubinstein 1990) 
$\frac{\partial c}{\partial t}=-\frac{\partial}{\partial x} J=\frac{\partial}{\partial x} D\left[\frac{\partial c}{\partial x}+\frac{z F c}{R T} \frac{\mathrm{d} \varphi}{\mathrm{d} x}\right]$.

Considering a fixed potential $\varphi$, this equation allows the evolution of the charge concentration to be calculated. However, a change in the charge concentration alters the electric potential, which needs to be recalculated to account for the change. Therefore, Eq. (7) must be supplemented by an equation for the electric potential $\varphi$, i.e., the Poisson equation (Rubinstein 1990; Griffiths 1999)

$\frac{\mathrm{d}^{2} \varphi}{\mathrm{d} x^{2}}=-\frac{\rho}{\varepsilon}$,

where $\varepsilon$ is the dielectric constant of the material (the product of the relative electric permittivity and the vacuum permittivity) and $\rho$ is the charge concentration, which can be obtained from the molar concentration after multiplying by the Faraday constant and $z$ as

$\frac{\mathrm{d}^{2} \varphi}{\mathrm{d} x^{2}}=-\frac{z F c}{\varepsilon}$.

Because the cellular environment is populated by multiple ionic species, the above equations should be generalized to the case of many ions. In this way, one ends up with $N$ diffusion equations of type (7) for each ionic species and one Poisson equation that takes into account all of the ions present in the environment, thus

$\frac{\partial c_{i}}{\partial t}=-\frac{\partial}{\partial x} J_{i}=\frac{\partial}{\partial x} D\left[\frac{\partial c_{i}}{\partial x}+\frac{z_{i} F c_{i}}{R T} \frac{\mathrm{d} \varphi}{\mathrm{d} x}\right], \quad i=1, \ldots, N$,

$\frac{\mathrm{d}^{2} \varphi}{\mathrm{d} x^{2}}=-\sum \frac{z_{i} F c_{i}}{\varepsilon}$.

Equations $(10,11)$ set the theoretical framework for the present research.

The data for the PNP model

To solve $(10,11)$ one must supplement them by the required parameters, including the ionic diffusion coefficients, the dielectric constant, and ionic concentrations. The main ion types present in a cell are $\mathrm{K}^{+}$and $\mathrm{Na}^{+}$ions.

Table 1 Intracellular concentrations of the charge carriers

After Lodish et al. (2008)

\begin{tabular}{ll}
\hline Ion & $\begin{array}{l}\text { Intracellular } \\
\text { concentration } \\
(\mathrm{mM})\end{array}$ \\
\hline $\mathrm{Na}^{+}$ & 12 \\
$\mathrm{~K}^{+}$ & 139 \\
Proteins $^{-}$ & 138 \\
$\mathrm{HCO}_{3}^{-}$ & 12 \\
\hline
\end{tabular}

The cell's negative charge originates mainly from proteins and $\mathrm{HCO}_{3}^{-}$. The corresponding concentrations are presented in Table 1.

As can be seen, the charge concentrations (which should in general be supplemented by the minor $\mathrm{Cl}^{-}$and $\mathrm{Mg}^{2+}$ concentrations) reduce almost to zero. This finding is consistent with the estimate of a capacitive charge held by the cell membrane. The specific capacitance of the cell membrane can be estimated as $C_{0}=1 \mu \mathrm{F} \mathrm{cm}^{-2}$ (Neher and Sakmann 1995), which results in a capacitance of a spherical cell with radius set to $R=16 \mu \mathrm{m}$ (the size of a Mat-Ly-Lu cell, rounded to a power of 2) equal to $C=4 \pi R^{2} C_{0}=32 \mathrm{pF}$. This capacitance at a voltage of $U=-90 \mathrm{mV}$ charges the membrane with $n=C U / e / N_{\mathrm{a}} 3 /$ $4 / \pi / R^{3}=0.002 \mathrm{mM}$ of (monovalent) negative charge ( $N_{\mathrm{a}}$ being the Avogadro number).

To simplify the model, I consider only charges due to proteins and $\mathrm{Na}^{+}, \mathrm{K}^{+}$, and $\mathrm{Ca}^{2+}$ ions, adjusting the protein charge to have the net charge of the cell consistent with the capacitive charge. The first two ionic species constitute most of the ions relevant to the process, and the $\mathrm{Ca}^{2+}$ ions, which are at very low concentration $(<0.0002 \mathrm{mM}$; Lodish et al. 2008), are included for predicting calcium dynamics, having a minor effect on the electric potential distribution.

The diffusion coefficients of the intracellular ions are given in Table 2. The proteins are assumed to be immobile in the first approximation. It can be noticed that the diffusion coefficient for the calcium ions is larger than for the entering sodium ions, allowing effective repulsion of calcium ions rather than their being "overtaken" by the sodium.

The dielectric constant is set close to the value in water, i.e., $\varepsilon_{0} \varepsilon_{\mathrm{r}}=10^{-9} \mathrm{~F} \mathrm{~m}^{-1}$.

The boundary conditions for the Poisson equation are set to be $\varphi(0)=0 \mathrm{mV}, \varphi(L)=-10 \mathrm{mV}$, where $L=32 \mu \mathrm{m}$ is the cell length. The voltage difference is set to reflect the electric field of $3 \mathrm{~V} \mathrm{~cm}^{-1}$, as used in Djamgoz et al. (2001). The boundary conditions for the Nernst-Planck equation are essentially set to zero flux at the boundaries (a special procedure is introduced to handle VGSC opening, as described below).

The initial condition for the Nernst-Planck equation is set to be the (unphysical) uniform distribution. To obtain the real ionic distribution, the Nernst-Planck (NP) equation is solved for $50 \mu \mathrm{s}$ (after which time the solution is

Table 2 Diffusion coefficients of the intracellular ions

\begin{tabular}{lr}
\hline Ion & Diffusion coefficient \\
\hline $\mathrm{Na}^{+}$ & $1.23 \times 10^{-10} \mathrm{~m}^{2} \mathrm{~s}^{-1}$ (Abelson 1949) \\
$\mathrm{K}^{+}$ & $17.3 \times 10^{-10} \mathrm{~m}^{2} \mathrm{~s}^{-1}$ (Hodgkin 1952) \\
$\mathrm{Ca}^{2+}$ & $5.3 \times 10^{-10} \mathrm{~m}^{2} \mathrm{~s}^{-1}$ (Donahue 1987) \\
\hline
\end{tabular}


essentially indistinguishable from a stationary solution of the NP equation). After that, a current pulse is applied for $1 \mathrm{~ms}$. The value of this current is set to depolarize the membrane to $0 \mathrm{mV}$; i.e., at each of the $N_{\mathrm{c}}$ time steps of the simulation corresponding to the interval of $1 \mathrm{~ms}$, a sodium concentration of $0.002 \mathrm{mM} N x / N_{\mathrm{c}}$ is injected at $x=0$ [keeping reflecting boundary conditions for the solution of the partial differential equation (PDE); Nx=32 is the number of grid points].

To investigate whether the assumption of a constantcurrent model of the VGSC activity limits the accuracy of the predictions (the actual sodium current has a pronounced spike), I modeled its action by a Dirac pulse. The conclusions were qualitatively similar, and the concentration changes at the cell boundaries were even more pronounced. However, since the real VGSC operates within about $1 \mathrm{~ms}$, and not the simulation time step of $10 \mathrm{ps,} \mathrm{I} \mathrm{present} \mathrm{the} \mathrm{results} \mathrm{for}$ the constant-current model of sodium inflow. The details of the numerical procedures applied to the solution of model equations are described shortly in the "Appendix".

\section{Results and discussion}

Figure 4 displays the rest-state distribution of the $\mathrm{Ca}^{2+}$ concentration. A similar chart can be obtained for the $\mathrm{Na}^{+}$ and $\mathrm{K}^{+}$ions, because in the rest state the cell exhibits a negative membrane potential and a negative intracellular charge. The unbalanced charge sticks to the membrane within the Debye length (Rubinstein 1990), contributing to a gradient in the ionic concentration. The Debye length in a cellular environment is of the order of $0.7 \mathrm{~nm}$ (Małysiak and Grzywna 2009), which cannot be directly reflected by the results on a lattice spacing of $1 \mu \mathrm{m}$, and a smaller spacing is impossible to apply due to computer time limitations. The

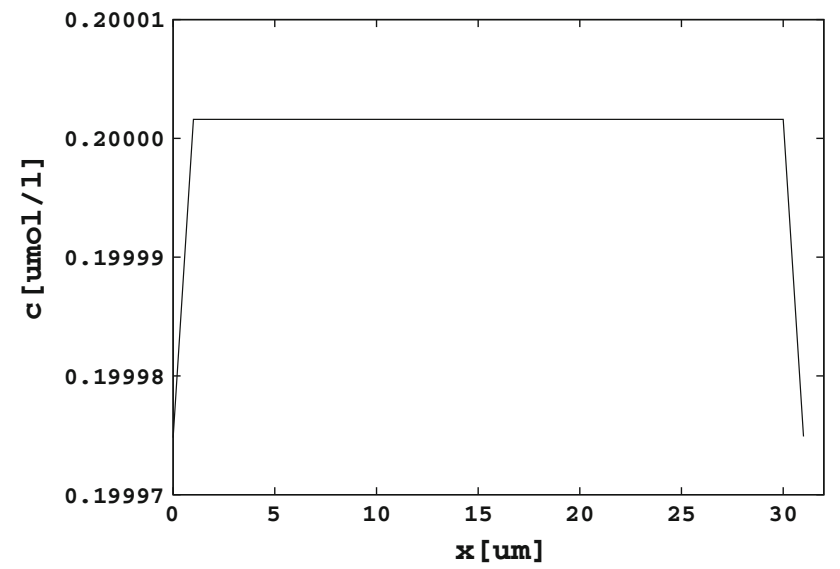

Fig. 4 An example of the rest-state distribution of ions. The calcium distribution is shown. Due to the negative membrane voltage in the rest state, the regions close to the membrane walls are depleted of ions. Similar plots can be obtained for $\mathrm{Na}^{+}$and $\mathrm{K}^{+}$ boundary concentration change shown in the chart is therefore averaged over $1 \mu \mathrm{m}$, and one should bear in mind that it is expected to be $1 \mu \mathrm{m} / 0.7 \mathrm{~nm}=1,429$ times larger within the Debye layer where the concentration can reach a value being $18 \%$ smaller than it is in the bulk concentration.

Starting at rest conditions, the $\mathrm{Na}^{+}$ions are injected into the cell domain, mimicking inflow at constant current, as shown in Fig. 5. In this figure one can see a linear increase in the $\mathrm{Na}^{+}$concentration at $x=0$, corresponding to the model of a constant sodium current. At this time resolution, diffusive effects are too slow to manifest and the concentration changes are linear with a slope reflecting the value of the current.

The $\mathrm{Ca}^{2+}$ concentration (as well as the $\mathrm{K}^{+}$concentration) reacts immediately to the increase in $\mathrm{Na}^{+}$at $x=0$, as shown in Fig. 6. The injected $\mathrm{Na}^{+}$ions repel the $\mathrm{Ca}^{2+}$ ions from the cathodal to the anodal side of the cell. This asymmetry holds even after time sufficient for diffusion to take place (Fig. 7).

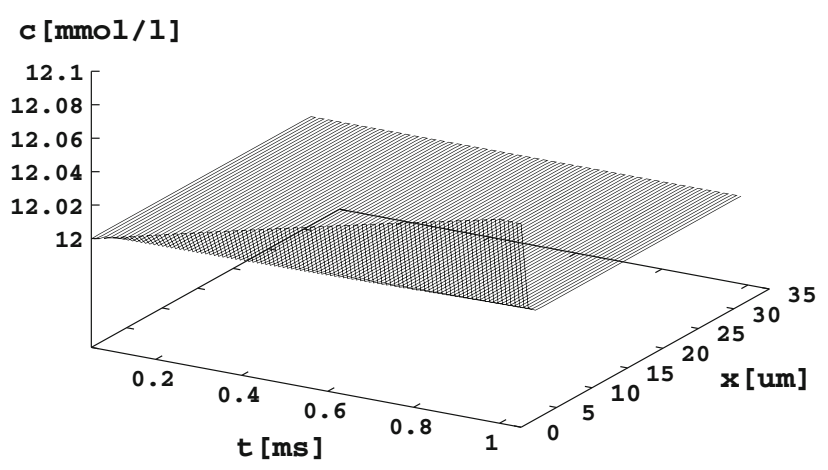

Fig. 5 The concentration of sodium ions during the activation of the sodium channels. This process is modeled by a constant current reflected by an almost linear slope of the concentration at $x=0$

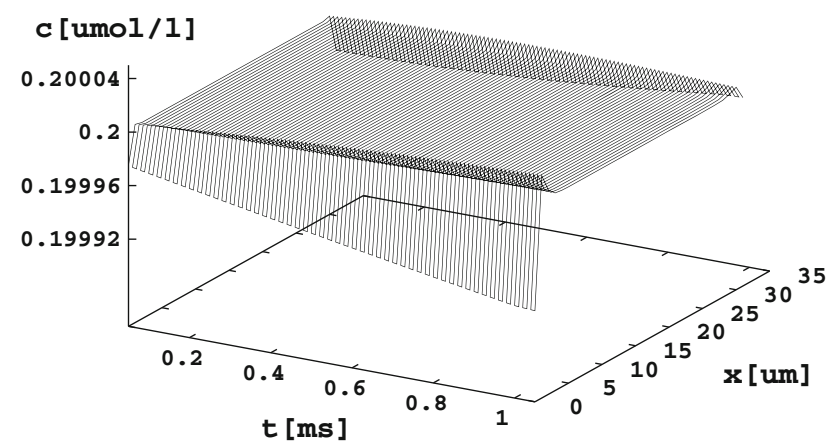

Fig. 6 The $\mathrm{Ca}^{2+}$ concentration immediately reacts to the changes in sodium concentration. The concentration at $x=0$ becomes decreased, and the concentration at $x=32 \mu \mathrm{m}$ increases. The interaction is very stiff, and relaxation effects are not seen in this model (although they can be seen after a Dirac sodium pulse at sufficient time resolution). Similar behavior is observed for the $\mathrm{K}^{+}$ concentration distribution 


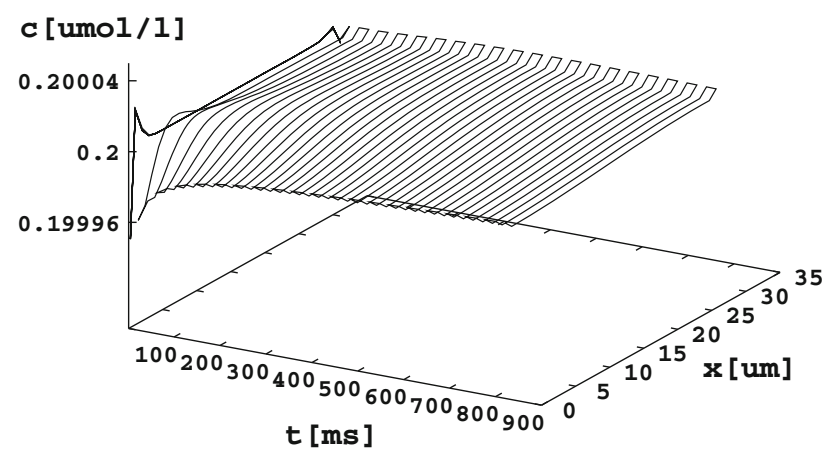

Fig. 7 Calcium concentration distribution for a longer time period. The asymmetric concentration change remains for a long time

The overall changes in the calcium concentration (Figs. 6, 7) seem to be small, but recalling the fact that they occur within the Debye length one can expect a change reaching a value of up to $57 \%$ of the bulk concentration, which supports the view that the inflow of $\mathrm{Na}^{+}$ions after cathodal depolarization may increase the anodal $\mathrm{Ca}^{2+}$ concentration (at the cost of the cathodal concentration). Such asymmetry may drive directional actin polymerization. In addition, as noted in the "Introduction," the action of the VGSCs may decrease the pericellular pH (Carrithers et al. 2007), which further speeds up actin polymerization (Wang et al. 1989) and can cause $\mathrm{Ca}^{2+}$ uptake (or a reduction in release) by internal stores (Willoughby et al. 2001). These effects would enhance the cathodal direction of galvanotaxis; however, after the whole membrane is depolarized, they could stop working. On the other hand, the asymmetry in the electrically repelled calcium should hold even after the depolarization reaches the anodal part, because the $\mathrm{Ca}^{2+}$ ions cannot go back to the cathodal part, which is already rich in positive charges.

The presented analysis fits very well with the behavior of the weakly metastatic AT-2 cells. These cells do not exhibit cathodal galvanotaxis because they do not upregulate VGSCs to contribute to the $\mathrm{Ca}^{2+}$ repulsion on the cathodal side. Instead, the internal $\mathrm{Ca}^{2+}$ ions (and other cations) drift in the applied DC field to increase the concentration on the cathodal side (Fig. 3), resulting in weakly directed motion with anodal orientation. The theory could, however, be questioned by stating that the leakage influx of the $\mathrm{Ca}^{2+}$ ions leads to anodal accumulation of $\mathrm{Ca}^{2+}$, as proposed in Cooper and Keller (1984). This requires deeper discussion.

Taking into account the leak $\mathrm{Ca}^{2+}$ permeability of a lipid membrane (between $8 \times 10^{-15} \mathrm{~cm} / \mathrm{s}$ and $2.5 \times$ $10^{-11} \mathrm{~cm} / \mathrm{s}$; Zeng et al. 1997) and considering a cell with radius $r=16 \mu \mathrm{m}$, one can calculate the currents corresponding to an intracellular $\mathrm{Ca}^{2+}$ concentration of $0.2 \mu \mathrm{M}$ and extracellular concentration of $1.8 \mathrm{mM}$ (Lodish et al. 2008). They range between $8.92 \times 10^{-20} \mathrm{~A}$ and
$2.78 \times 10^{-16}$ A. Fitting the conductance according to the relation $I=g_{\text {Ca,leak }}\left(U-U_{\mathrm{Ca}}\right)$, where $U$ is the rest membrane potential and $U_{\mathrm{Ca}}$ is the Nernst potential for $\mathrm{Ca}^{2+}$, and normalizing to the cell's surface, one obtains $g_{\text {Ca,leak }}$ varying between $13.2 \mathrm{fS} / \mathrm{cm}^{2}$ and $41.24 \mathrm{pS} / \mathrm{cm}^{2}$. With these data it is possible to calculate the increment of the leak $\mathrm{Ca}^{2+}$ current, associated with a DC field, on a model cubic cell with side length of $26 \mu \mathrm{m}$ (the volume of such a cell being equal to the volume of the considered cells). With a DC field of $3 \mathrm{~V} / \mathrm{cm}$, the anodal membrane hyperpolarizes by about $5 \mathrm{mV}$, which results in a current change between $4.46 \times 10^{-22} \mathrm{~A}$ and $1.39 \times 10^{-18} \mathrm{~A}$. This current could lead to accumulation of $\mathrm{Ca}^{2+}$ provided that the cytoplasmic current is lower and cannot account for such contribution. The cytoplasmic current can be estimated by considering the membrane resistance after Kager et al. (2000), equal to $75 \pm 25 \mathrm{M} \Omega$ for a cell with surface of $1,686 \mu \mathrm{m}^{2}$. The surface of the cubic face of the cubic cell is $676 \mu \mathrm{m}^{2}$, and consequently, the membrane resistance of this face is higher, i.e., $187 \mathrm{M} \Omega$. The resistance of the cubic cell's cytoplasm is taken as $240 \mathrm{k} \Omega$ after Stinstra et al. (2005), who reported cytoplasm conductance of $0.16 \mathrm{~S} / \mathrm{m}$. Calculating the voltage divided across the membrane-cytoplasm-membrane resistances, one obtains a voltage drop for the cytoplasm of $12.8 \mu \mathrm{V}$. By Eq. (5), this predicts a cytoplasmic calcium current of $0.52 \mathrm{fA}$, larger than the increase in the leak influx. This indicates that the cytoplasm layers close to the membrane will actually become depleted of $\mathrm{Ca}^{2+}$.

The anodal leak accumulation of $\mathrm{Ca}^{2+}$ can however occur in some conditions. Such conditions may arise in some cells, for example, muscular cells, which are equipped with $\mathrm{Ca}^{2+}$ leak channels (Obejero-Paz et al. 1998). In this case, the rest-state leak current can take large values, of the order of $0.36 \mathrm{pA}$, which results in conductance of $0.21 \mu \mathrm{S} / \mathrm{cm}^{2}$ (A7r5 cell diameter $\sim 16 \mu \mathrm{m}$ ), giving membrane current contributions due to the DC field too large to be compensated by the cytoplasmic current $(7.2 \mathrm{fA})$. In such a case, the concentration of $\mathrm{Ca}^{2+}$ would increase locally (probably at the cost of $\mathrm{K}^{+}$ions to keep the voltage unaltered) until the PMCA pump activates (due to the increased $\left[\mathrm{Ca}^{2+}\right]$ ) to a level sufficient to counterbalance this leak.

To discuss how the proposed mechanism fails to describe the VGSC-dependent anodal (not cathodal) galvanotaxis of MDA-MB-231 breast cancer cells (Fraser et al. 2005; Isbilen et al. 2006), one needs to consider mechanisms other than cathodal repulsion of $\mathrm{Ca}^{2+}$. One of the explanations could be a possible action of the VGCCs, but the literature states that the breast cancer cells do not possess VGCCs (Roger et al. 2004). Second, the breast cancer upregulates the plasma membrane $\mathrm{Ca}^{2+}$ (PMCA) pumps (Carafoli and Brini 2007; Monteith et al. 2007). 
Such pumps serve to decrease the intracellular calcium within the cell and could make the calcium concentration insufficient to influence the gelsolin-based blocking of actin polymerization, leaving only the $\mathrm{pH}$ dependency resulting from the VGSC activation (Wang et al. 1989; Carrithers et al. 2007). This, however, would imply cathodal galvanotaxis and cannot explain the anodal direction of motion. A final option could be that the NCX exchanger enters into the action [there is no literature evidence that it is absent in MDA-MB-231, and a good suggestion of its presence may be found in Winnicka et al. (2007)]. Such an exchanger could stop removing calcium from the cathodal side after depolarization [intracellular $\mathrm{Na}^{+}$blocks the exchanger (Hilge et al. 2007)], opposing the repulsive effect in the decrease of the cathodal $\mathrm{Ca}^{2+}$ concentration.

\section{Conclusions}

In this work, I show that the action of the VGSCs can cause repulsion of calcium ions from the cathodal side of the cell to the anodal side. In this way, an asymmetry in the calcium concentration can arise close to the membrane. It seems that such asymmetry is just what is needed to explain the VGSC-dependent cathodal galvanotaxis, which requires depletion of calcium on the cathodal side to cause actin polymerization and enrichment on the anodal side to cause myosin contraction.

What is important is that the presented theory requires a time delay between the depolarization events on the cathodal and on the anodal side of the cell. Such a time delay can also be used to propose directionality in other mechanisms acting on calcium dynamics, such as VGSC-mediated $\mathrm{pH}$-induced internal store activity or facilitation of actin polymerization, or (in the case of cells that have them) in the operation of NCX exchangers.

The asymmetry in the calcium distribution remains even after diffusion starts to manifest, which suggests that the effect may hold for a longer period and may accumulate in subsequent depolarization cycles. It is not expected to be destroyed by the proceeding depolarization of the anodal part of the membrane, since the cathodal part already contains a large (repulsive) charge by that time, preventing any acceptance of additional charge. The asymmetry, in case of a real cell, can show even larger values of calcium concentration difference, because the sodium current reveals a spike. All this supports the qualitative model of galvanotaxis sketched in this paper, where the interplay between sodium and calcium ions is taken as the main contribution.

Importantly, the model not only explains the galvanotaxis of the highly metastatic prostate Mat-Ly-Lu cells but also accounts for the reduced mobility of AT- 2 cells, and leaves enough space for the anodal galvanotaxis of breast cancer cells.

Open Access This article is distributed under the terms of the Creative Commons Attribution License which permits any use, distribution, and reproduction in any medium, provided the original author(s) and the source are credited.

\section{Appendix: the solution method of the NP equation}

The Nernst-Planck equation is a nonlinear drift-diffusion equation, which causes stability problems during numerical integration. To work around this problem, I have taken the approach of an explicit scheme with stabilization method, i.e., the explicit upwind scheme ( $\mathrm{Ni}$ et al. 1998) applied to the drift term of the equation. The upwind scheme takes the following approximation to the first-order derivative of concentration:

$\frac{\mathrm{d} c}{\mathrm{~d} x} \approx\left\{\begin{array}{ll}(c[x+h]-c[x]) / h: & \frac{\mathrm{d} \varphi}{\mathrm{d} x} \geq 0 \\ (c[x]-c[x-h]) / h: & \frac{\mathrm{d} \varphi}{\mathrm{d} x}<0\end{array}\right\}$,

where the potential derivative is taken as a central difference. The time step for the simulation depends upon the gradient of the potential and initially was taken to be equal to $10 \mathrm{ps}$, after $1.1 \mathrm{~ms}$ being increased to $100 \mathrm{ps}$ (further increases are possible but were not used in this research).

The second-order derivative was calculated as a central difference in a standard way. This method allowed results to be obtained for reasonable dielectric constants $\left(\epsilon_{r}=\right.$ 100) while alternative approaches such as the CrankNicolson implicit scheme became unstable at dielectric constant values several orders of magnitude weaker.

\section{References}

Abelson PH, Duryee WR (1949) Radioactive sodium permeability and exchange in frog eggs. Biol Bull 96:205-217

Berg HC (1982) Random walks in biology. Princeton University Press, Princeton

Borys P (2008) On the galvanotaxis of prostate cancer cells. Gliwice Scientific Meetings Conference, poster 3

Bray D (2001) Cell movements: from molecules to motility. Garland, New York

Carafoli E, Brini M (eds) (2007) Calcium signaling and disease. Springer, New York

Carrithers MD, Dib-Hajj S, Carrithers LM, Tokmoulina G, Pypaert M, Jonas EA, Waxman SG (2007) Expression of the voltagegated sodium channel $\mathrm{Na}$ V1.5 in the macrophage late endosome regulates endosomal acidification. J Immunol 178(7822):7832

Coalson RD, Kurnikova MG (2007) Poisson-Nernst-Planck theory of ion permeation through biological channels. In: Chung $\mathrm{SH}$, Andersen OS, Krishnamurthy V (eds) Biological membrane ion channels. Springer, New York 
Cooper MS, Keller RE (1984) Perpendicular orientation and directional migration of amphibian neural crest cells in dc electrical fields. Proc Natl Acad Sci USA 81:160-164

Djamgoz MBA, Mycielska M, Madeja Z, Fraser SP, Korohoda W (2001) Directional movement of rat prostate cancer cells in direct current electric field: involvement of voltage-gated $\mathrm{Na}+$ channel activity. J Cell Sci 114:2697-2705

Donahue BS, Abercrombie RF (1987) Free diffusion coefficient of ionic calcium in cytoplasm. Cell Calcium 8:437-448

Fraser SP, Diss JKJ, Chioni AM, Mycielska ME, Pan H, Yamaci RF, Pani F, Siwy Z, Krasowska M, Grzywna Z, Brackenbury WJ, Theodorou D, Koyuturk M, Kaya H, Battaloglu E, De Bella MT, Slade MJ, Tolhurst R, Palmieri C, Jiang J, Latchman DS, Coombes RC, Djamgoz MBA (2005) Voltage-gated sodium channel expression and potentiation of human breast cancer metastasis. Clin Cancer Res 11:5381-5389

Griffiths DJ (1999) Introduction to electrodynamics. Prentice Hall, New Jersey

Grimes JA, Fraser SP, Stephens GJ, Dowing JEG, Laniado ME, Foster CS, Abel PD, Djamgoz MBA (1995) Differential expression of voltage-activated $\mathrm{Na}+$ currents in two prostatic tumor cell lines: contribution to invasiveness in vitro. FEBS Lett 369:290-294

Hilge M, Aelen J, Perrakis A, Vuister GW (2007) Structural basis for $\mathrm{Ca}^{2+}$ regulation in the $\mathrm{Na}^{+} / \mathrm{Ca}^{2+}$ exchanger. Ann NY Acad Sci 1099:7-15

Hodgkin AL, Keynes RD (1952) The mobility and diffusion coefficient of potassium in giant axons from Sepia. J Physiol 119:513-528

Isbilen B, Fraser SP, Djamgoz MBA (2006) Docosahexaenoic acid (omega-3) blocks voltage-gated sodium channel activity and migration of MDA-MB-231 human breast cancer cells. Int J Biochem Cell Biol 38:2173-2182

Kager H, Wadman WJ, Somjen GG (2000) Simulated seizures and spreading depression in a neuron model incorporating interstitial space and ion concentrations. J Neurophysiol 84:495-512

Kurnikova MG, Coalson RD, Graf P, Nitzan A (1999) A lattice relaxation algorithm for three-dimensional poisson-NernstPlanck theory with application to ion transport through the Gramicidin A channel. Biophys J 76:642-656

Lodish HF, Berk A, Kaiser CHA, Krieger M, Scott MP, Bretscher A, Ploegh H (2008) Molecular cell biology. W.H. Freeman, New York

Małysiak K, Grzywna ZJ (2009) Electrostatic interactions during Kv1.2 N-type inactivation: random-walk simulation. Eur Biophys J 38:1003-1012
Monteith GR, Andrew DM, Faddy HM, Roberts-Thomson SJ (2007) Calcium and cancer: targeting $\mathrm{Ca}^{2+}$ transport. Nat Rev 7:519-530

Mycielska ME, Djamgoz MBA (2004) Cellular mechanisms of directcurrent electric field effects: galvanotaxis and metastatic disease. J Cell Sci 117:1631-1639

Neher E, Sakmann B (1995) Single channel recording. Plenum, New York

Ni MJ, Tao WQ, Wang SJ (1998) Stability-controllable second-order difference scheme for convection term. J Therm Sci 7:119-130

Obejero-Paz CA, Jones SW, Scarpa A (1998) Multiple channels mediate calcium leakage in the A7r5 smooth muscle-derived cell line. Biophys J 75(1271):1286

Risken H (1989) The Fokker-Planck equation: methods of solution and applications. Springer, Berlin

Roger S, Le Guennec JY, Besson P (2004) Particular sensitivity to calcium channel blockers of the fast inward voltage dependent sodium current involved in the invasive properties of a metastatic breast cancer cell line. Br J Pharmacol 141:610-615

Rubinstein I (1990) Electro-diffusion of ions. SIAM Studies in Applied Mathematics, Philadelphia

Siwy Z, Mycielska ME, Djamgoz MBA (2003) Statistical and fractal analyses of rat prostate cancer cell motility in a direct current electric field: comparison of strongly and weakly metastatic cells. Eur Biophys J 32:12-21

Stinstra JG, Hopenfeld B, Macleod RS (2005) On the passive cardiac conductivity. Ann Biomed Eng 33:1743-1751

Wang F, Sampogna RV, Ware BR (1989) pH dependence of actin self assembly. Biophys J 55:293-296

Willoughby D, Thomas RC, Schweining CJ (2001) The effects of intracellular $\mathrm{pH}$ changes on resting cytosolic calcium in voltageclamped snail neurones. J Physiol 530:405-416

Winnicka K, Bieławski K, Bieławska A, Miltyk W (2007) Apoptosismediated cytotoxicity of ouabain, digoxin and proscillaridin $\mathrm{A}$ in the estrogen independent MDA-MB-231 breast cancer cells. Arch Pharm Res 30(1216):1224

Yan X, Han J, Zhang Z, Wang J, Cheng Q, Gao K, Ni Y, Wang Y (2009) Lung Cancer A549 cells migrate directionally in DC electric fields with polarized and activated EGFRs. Bioelectromagnetics 30:29-35

Zeng J, Borchman D, Paterson CA (1997) Calcium permeability in large unilamellar vesicles prepared from bovine lens cortical lipids. Exp Eye Res 64:115-120 